\title{
Understanding the Role of Ethnic Density Effect: Issues of Acculturation, Discrimination and Social Support
}

\author{
Tomas Jurcik ${ }^{1}$, Rana Ahmed ${ }^{1}$, Esther Yakobov ${ }^{1}$, Ielyzaveta Solopieieva ${ }^{1}$, and Andrew G. Ryder ${ }^{1,2}$ \\ ${ }^{1}$ Concordia University \\ ${ }^{2}$ Jewish General Hospial
}

\begin{abstract}
Ecological factors in psychological acculturation research are often neglected, although recent work suggests that context and acculturation may interact in predicting adaptation outcomes. The ethnic density effect-the protective effect related to a greater proportion of people from the same ethnic group living in a particular neighborhood - might be one such ecological candidate. The current study integrates these constructs by unpacking the perceived ethnic density effect and examining how it is related to acculturation in a diverse sample $(\mathrm{N}=146)$ of immigrant students in Montreal, Canada. It was found that the negative relation between perceived ethnic density and depression was mediated by discrimination but not by social support. Furthermore, a crossover interaction indicated that heritage acculturation was protective against depression for those residing in ethnically concentrated neighborhoods but not for those living in ethnically sparse neighborhoods. This strongly supports an ecology-acculturation fit, highlighting the need to contextualize acculturation research. (c)2013 Wiley Periodicals, Inc.
\end{abstract}

Most psychologists would agree with the statement that immigrants acculturate and function within a sociocultural context. While this may seem like an empty truism, relatively little continues to be known about the interplay between ecological and cultural-level phenomena in the clinical manifestations of disorder. Ecological factors such the ethnic composition of neighborhoods may confer protection as well as risk, and differentially favor certain acculturation styles over others. The purpose of the current study is to further integrate the findings related to neighborhood ecology, more specifically, perceived ethnic density, with findings obtained in the cultural psychological literatures related to acculturation, discrimination, and social support in immigrants.

The research described in this article was supported by an FQRSC doctoral award to Tomas Jurcik and an FRSQ chercheur-boursier award to Andrew G. Ryder. This research was conducted as part of Tomas Jurcik's doctoral dissertation at Concordia University, supervised by Andrew G. Ryder. We thank Anne Lam for her assistance with data cleaning, Dr. Edison Trickett for suggesting pertinent references, Jessica Dere for her helpful comments on the analyses, as well as Catherine Malboeuf-Hurtubise and other members of the Culture, Health and Personality Lab at Concordia University for their general support. Please address correspondence to: Tomas Jurcik and/or Andrew Ryder, 7141 Sherbrooke St. W., Montreal, Quebec, H4B 1R6, Canada. E-mail: t_jurciklive.concordia.ca and/or andrew.ryderconcordia.ca

\section{Unanswered Questions in Acculturation Research}

Numerous researchers have attempted to understand how people experience cultural and psychological change through their interaction with other cultural groups, commonly known as acculturation, and how such processes may be related to mental health in immigrants (e.g., Sam, 2006). However, controversial conceptual and operational issues continue to plague acculturation research (Rudmin, 2003, 2009). This is not surprising, given that there is no consistent definition of culture, and researchers continue to grapple with measuring multiple aspects related to acculturation: a sending/heritage culture, a receiving/mainstream one, and possibly even a new or complex emergent identities (Ryder \& Dere, 2010). Thus, some researchers view acculturation along one dimension, known as the unidimensional model (e.g., Gordon, 1964; Suinn, Abona, \& Khoo, 1992), others view it along two orthogonal dimensions (e.g., Costigan \& Su, 2004; Ryder, Alden, \& Paulhus, 2000), and more complex models exist measuring acculturation along three or more dimensions (Flannery, Reise, \& Yu, 2001). While less research exists on the latter approach, bidimensional acculturation models have shown greater utility than unidimensional ones. In other words, examining heritage and mainstream dimensions independently yields greater predictive validity than pitting heritage versus mainstream acculturation as polar opposites on a continuum (Ryder et al., 2000). Mounting evidence has accumulated to date showing the superiority of bidimensional models over unidimensional ones, despite some limitations (Schwartz, Unger, Zambaonga, \& Szapocznik, 2010). 
Operationally speaking, bidimensionl acculturation is the degree to which a bicultural person (e.g., immigrant) identifies with the behavior, beliefs, and values of the heritage and/or the predominant mainstream cultural groups (Ryder et al., 2000). Although these two dimensions are considered to be statistically independent (e.g., Costigan \& Su, 2004; Ryder et al., 2000), people may identify with both cultural groups simultaneously (Berry, 2006a). Immigrants are presumed to select or navigate between cultural identities, often referred to as acculturation strategies (Berry, 2006a). Those who more closely endorse a mainstream cultural identity (Ryder et al., 2000; Zhang, Mandl, \& Wang, 2010), or both heritage and mainstream cultural identities (Berry, 2006a), show better psychological adjustment outcomes. However, the pattern of findings has not been consistent empirically across cultural and demographic groups. For example, it is conceivable that older adult migrants could differ from younger people in what acculturation strategies are most adaptive to their well-being (Schwartz et al., 2010).

Concurrently, the attitude of minority cultural group members towards the larger society, and that society's policies and attitudes towards migration, may all influence acculturation strategies (Berry, 1997; Berry, 2006b). For example, a pluralistic policy of multiculturalism may encourage identification with both mainstream and heritage cultures, while a segregationist approach might foster or even demand maintenance of a heritage cultural identity (see Berry, 2006b). Inconsistent findings between level of acculturation and adjustment outcomes among different ethnic/immigrant groups and settings have also raised questions about how community-level contextual factors, such as neighborhood ethnic composition, might interact with acculturation (e.g., Birman, Trickett, \& Buchanan, 2005; Schwartz et al., 2010). Thus, the relation between acculturation and adjustment outcomes are far from simple according to the theoretical and empirical literature: Some groups may benefit using strategies that may be irrelevant or even problematic to others. The term acculturation strategies implies voluntary control, but they at least partly depend on the sociopolitical and ecological setting into which migrants arrive, and in some cases may be outside of personal control (Schwartz et al., 2010).

For instance, mainstream but not heritage acculturation was found to play a role in protecting people from depressive symptoms in groups of ethnic Chinese in Vancouver (Ryder et al., 2000) and Germany (Zhang et al., 2010), but the reverse was found recently, with heritage (but not mainstream) acculturation predicting less distress in a visible minority group in Montreal (Ahmed, Jurcik \& Ryder, 2011). Explanations for such differences remain elusive. One possibility is that Vancouver and Montreal are culturally and linguistically different population centers, with Montreal showing more ethnic segregation according to census tract analysis compared with Vancouver (see Balakrishnan, Maxim,
\& Jurdi, 2005). Thus, heritage acculturation may play a larger role with more recent immigrants in contexts where multiple mainstream groups exist and cultural segregation is more pronounced, as in Montreal, which already includes two mainstream cultural groups-English and French Canadian (Bourhis, Montaruli, Geledi, Harvey, \& Barrette, 2010). In turn, mainstream acculturation may be more relevant to mental health in other, perhaps more established minority groups, or in less segregated contexts, such as the ethnic Chinese in Vancouver. In sum, the inconsistent findings in acculturation research imply that greater attention to context is warranted.

\section{Ethnic Density Effect: Waiting to Be Explained}

Related clues as to how context may matter to acculturation come from findings obtained in the social psychiatry and epidemiology literatures. One ecological factor that has been receiving increasing interest over the last two decades has been the ethnic density effect (e.g., Whitley, Prince, McKenzie, \& Stewart, 2006). The ethnic density (ED) effect relates to the benefits conferred on those who live in neighborhoods where there is a greater proportion of individuals from the same ethnic background. Although a complex construct, ethnicity is operationalized broadly in this research, often by country of origin (e.g., Pakistan), region and racial group (e.g., Black Caribbean), or along linguistic lines (e.g., Russian speakers). ED can be measured objectively (e.g., using actuarial census data) or subjectively (i.e., perceived density), with the two approaches yielding moderately correlated indices (Stafford, Bécares, \& Nazroo, 2009). Although it has to date been studied mostly at the objective level, there is recent interest in examining how perceived ED may also be associated with health (Stafford et al., 2009).

This negative relation between ethnic concentration and disorder has recently been obtained with various immigrant groups studied in the United Kingdom (e.g., South Asian, Caribbean, and Irish groups; Das-Munshi, Becares, Dewey, Stansfeld, \& Prince, 2010; Stafford, Becares, \& Nazroo, 2010). Specifically, ED has been associated with fewer common mental health disorders (Das-Munshi et al., 2010), fewer psychotic disorders (Boydell et al., 2001), lower rates of alcohol abuse (Becares, Nazroo, \& Stafford, 2011), with mixed findings for long-term illness limiting a person's daily activities (Stafford et al., 2009). In the Canadian context, Malzberg (1964) found that English Canadians living in French-majority neighborhoods were at increased risk of being hospitalized for a psychotic disorder compared with French Canadians; the same trend was found for French Canadians where they formed the minority group.

More recently, overall immigrant density, a related concept, has been shown to protect Canadian visible minority migrants from depression (Stafford et al., 2010). The relation between ethnic or immigrant concentration and out- 
comes seems to be linear for the most part (e.g., Becares, Nazroo, \& Stafford, 2009; Boydell et al., 2001; Stafford et al., 2010), although curvilinear relationships have also been obtained (Neeleman, Wilson-Jones, \& Wessely, 2001). On some occasions, greater ethnic density has been linked to poorer outcomes, but this seems to be less common. For example, although Becares and colleagues (2009) noted that Bangladeshi and Indian participants reported lower rates of psychotic symptoms in more concentrated neighborhoods, the reverse was found for Pakistani participants.

Despite nearly eight decades of research, the mechanisms of the ethnic density effect remain unclear (Das-Munshi et al., 2010; Whitley et al., 2006). The term itself may be misleading because studies are generally correlational and thus cannot clearly unravel cause from effect. Nevertheless, ED has been related to decreased discrimination and/or increased social support (Becares et al., 2009; Das-Munshi, 2010; Stafford et al., 2010; Whitley et al., 2006; but for a paradoxical finding see Birman et al., 2005), suggesting the possibility that the effects of ethnic density may operate indirectly through such variables. Thus, immigrants living in more concentrated neighborhoods may encounter less hostility from other groups while also having increased opportunities for various forms of social support. In other words, ethnic density may allow for an increased access to resources and the formation of a larger ethnic in-group, thereby providing a "psychic shelter" from the discrimination of the majority group (Whitley et al., 2006). Having a greater proportion of co-migrants may also ease the stress of adaptation into a new environment (cf. Stafford, Newbold, \& Ross, 2011). These potential explanations are consistent with a large and extant body of empirical findings that have linked depression in migrants and minority groups to increased discrimination (Jung, Hecht, \& Wadsworth, 2007; Noh \& Kaspar, 2003) and lack of social support (see review by Mirsky, 2009). Thus, the relation between ethnic density and fewer symptoms may operate indirectly through increased social support and decreased discrimination.

Recently, Das-Munshi et al. (2010) attempted to test the discrimination and social support mediation hypothesis for ethnic density but did not obtain an indirect effect. This may, however, have been due to limitations in measurement. Despite using a sophisticated sampling design culling random samples of various ethnic groups, both discrimination and social networks were measured with relatively few categorical items, and participants were dichotomized into groups of people who either had or did not have common mental disorders. Furthermore, objective rather than subjective density was used. Studies comparing objective to subjective measures have also demonstrated predictive advantages for the latter. For example, subjective measures of socioeconomic status are better indicators of health status than objective ones (Singh-Manoux, Mormot, \& Adler, 2005) and neighborhood perceptions are more strongly related to distress than neighborhood location (Christie-Mizell, Steelman, \& Jennifer, 2003). By extrapolation, perceived ethnic density may correlate more strongly or more consistently with various other subjective outcomes, such as distress, perceived discrimination, and social support experiences, than objective measures.

\section{Acculturation Meets Ethnic Density}

Political factors and attitudes of the majority culture may moderate acculturation (Berry, 2006b). However, it is possible that more microlevel environments such as neighborhoods also interact with individual acculturation orientations (Birman et al., 2005). Recently, Birman and colleagues (e.g., Birman et al., 2005; Miller et al., 2009) have been examining the link between culture and neighborhood-level factors in Illinois. Their findings suggest that acculturation levels may interact with immigrant density. For example, Miller and colleagues (2009) found that alienation from American culture in a group of older Russian women migrants in the United States who showed high levels of Russian behavioral acculturation (i.e., observable lifestyle behaviors) was attenuated if these individuals lived in neighbourhoods with a higher concentration of immigrants. In other words, if Russian behavior took place in an area with a high proportion of immigrants, then its association with alienation was not as pronounced. It is possible that these women felt more comfortable expressing Russian ways of behaving in neighborhoods where there were more immigrants in general.

These findings beg the question of whether the protective effect of heritage acculturation on depression in Montreal (Ahmed et al., 2011) may be enhanced in neighborhood contexts of higher ethnic density. Such a finding would be suggestive of an ecology acculturation fit or match (Miller et al., 2009; Schwartz et al., 2010), where the benefits of high heritage acculturation may be further enhanced in ethnically concentrated versus sparse neighborhoods. That is, immigrants living in a milieu that is synchronous to their cultural values are likely to benefit the most (i.e., show the least amount of distress). In contrast, if the neighborhood environment does not support their heritage culture identification, heritage acculturation-mental health benefits may be less pronounced. Furthermore, thus far the ecology-acculturation research has focused on objective rather than subjective ethnic or immigrant density and has not yet examined mental health (e.g., depression) outcomes. While the mechanisms of the ethnic density effect remain elusive, the interaction between acculturation and ethnic density has also received only very limited research attention to date.

\section{Aims and Hypotheses}

The current study sought to determine whether a negative relation between perceived ethnic density and depression ex- 
ists, and whether it can be explained by discrimination and social support experiences in a group of immigrant University students in Montreal. It also attempted to test whether perceived ethnic density might interact with heritage acculturation for depression, that is, whether perceived ethnic density might enhance the protective relation between heritage acculturation and depression. Given the heterogeneity of the current immigrant sample, objective comparison measures of ethnic density were not examined.

Three sets of hypotheses were derived based on the literature review and previous findings from pilot studies conducted by members of our research group.

H1. (a) Perceived ethnic density was expected to be related negatively to depression, (b) negatively to perceived discrimination, and (c) positively to social support. (d) Perceived discrimination was anticipated to relate positively to depression, whereas (e) heritage acculturation was anticipated to be related negatively to depression, and (f) social support was also hypothesized to relate negatively to depression.

H2. (a) Lower discrimination and (b) higher social support were expected to mediate the negative relation between perceived ethnic density and depression.

H3. The relation between greater heritage acculturation and lower depression was expected to be amplified if ethnic density was perceived to be high.

A series of exploratory analyses were run to examine additional potential perceived ED by mainstream acculturation, ED by social support, and ED by discrimination interactions on depression, because it is possible that ethnic density might mitigate or augment effects related to acculturation, discrimination and social support.

\section{Method}

\section{Participants}

The final sample yielded 146 immigrant university student participants (see Table 1 for demographics). The mean age was 26, and the length of time in Québec was approximately 9.5 years, with most participants $(78 \%)$ having lived in Québec longer than in the rest of Canada. Participants were retained if they identified as first generation immigrants to Canada. Nonimmigrant students (including those born in Canada and those on a student visa), immigrants from the United States or those who had at least one parent from Canada or the United States were excluded from the study, along with one participant who reported that his responses did not reflect his experience.

\section{Procedure}

University student participants at a large English-language university in Montreal, Québec completed an extensive online survey between 2010 and 2012 on adjustment to life in Canada. The study was approved by the institutional review board and participants consented to the study online. Participants either received course credit for an undergraduate psychology course or could win $\$ 50$ if not enrolled in a credit-granting course. The study was advertised in the psychology department, on the two university campuses, and by various cultural and immigrant student associations.

\section{Measures}

Perceived ethnic density (variation of Stafford et al., 2009). Perceived ED was measured using one item to estimate of the participants' neighborhood ethnic concentration. The directions to participants were to estimate how many people from their own ethnic group live in their neighborhood on a 5-point scale, ranging from 1 (none or hardly any) to 5 (almost all or all of the neighborhood). The participants were given examples of traditional names of Montreal neighborhoods for definition and orientation purposes. The distribution of responses was positively skewed (Table 1), with a majority of participants reporting that they lived in neighborhoods with none or almost none $(37.1 \%)$ or only some of the neighborhood $(50.3 \%)$ containing their ethnic group. Given the considerable diversity of national groups, this suggests that participants perceived their minority status fairly accurately (list of origin countries available upon request from the authors; see also regional origin in Table 1).

Vancouver Index of Acculturation (VIA; Ryder et al., 2000). The VIA assesses heritage and mainstream acculturation (adapted to the French-Canadian context) on two independent subscales - 10 items each measuring identical phenomena using a 9-point Likert scale, ranging from 1 (strongly disagree) to 9 (strongly agree). Both subscales are considered to be conceptually and empirically orthogonal (Ryder et al., 2000). A sample heritage item includes "I often participate in heritage culture traditions" and a mainstream item includes "I believe in mainstream French-Canadian values." Heritage and mainstream acculturation subscale scores showed good internal reliability coefficients $(\alpha=.89$ and .86 , respectively). French-Canadian acculturation was used as the default mainstream culture. The official language of Québec is French, and because Québec immigration policies promote French language and Québécois culture in newcomers (referred to as la francisation des Immigrants; Immigration et Communautés Culturelles Québec, 2012), many of these students would have attended secondary school or (pre-University) college in French, although they were currently studying at an English-language institution. 
Table 1. Demographic Variables for First Generation University Student Immigrants to Canada $(N=146)$

\begin{tabular}{lr}
\hline Variables & \\
\hline Gender (\% female) & 85.6 \\
Age (years): Mean $(S D)$ & $26.01(7.53)$ \\
Canada (years): Mean $(S D)$ & $11.50(7.31)$ \\
Québec (years) ${ }^{\mathrm{a}}$ : Mean $(S D)$ & $9.54(7.13)$ \\
Region of birth (\%) & 29.5 \\
Europe & 25.3 \\
Middle East and North Africa & 15.8 \\
East and Southeast Asia & 15.1 \\
Latin America & 8.2 \\
South Asia & 4.1 \\
Caribbean & 2.1 \\
Africa (sub-Saharan) & \\
Perceived ethnic density ${ }^{\mathrm{b}}(\%)$ & 37.1 \\
None or hardly none & 50.3 \\
Some & 7.7 \\
About half & 4.2 \\
More than half & 0.7 \\
All or almost all & \\
\hline
\end{tabular}

Note. $\mathrm{SD}=$ standard deviation.

${ }^{\text {a }} 78.1 \%$ lived longer in Québec than the rest of Canada; U.S. immigrants and those with Canadian parents were excluded.

${ }^{\mathrm{b}} N=143$.

Multidimensional Scale of Perceived Social Support (MSPSS; Zimet, Dahlem, Zimet, \& Farley, 1998). The MSPSS is a 12-item measure assessing subjective social support in the domain of family, friends, and significant others on a 7-point Likert scale, ranging from 1 (very strongly disagree) to 7 (very strongly agree). A sample item includes "My friends really try to help me." It has been used previously in other studies examining the relation between social support and depression in immigrants (e.g., Ritsner, Ponizovsky, \& Ginath, 1997). Internal reliability was considered to be high in the current sample $(\alpha=.94)$.

Perceived Discrimination Scale (PERDS; Noh \& Kaspar, 2003). The PERDS is an eight item measure used to assess subjective experiences related to harassment, specifically in Canada, on a 5-point scale, ranging from 1 (never) to 5 (all the time). A sample item includes "In Canada, because of discrimination, have you ever been hit or handled roughly?" The measure has been associated with depression in immigrants (Noh \& Kaspar, 2003) and in the current study it showed adequate internal reliability $(\alpha=.83)$.

Center for Epidemiologic Studies_Depression (CES-D;
Radloff, 1977). The CES-D is a 20-item measure assessing depressive symptoms over the last week on a 4-point scale, ranging from 1 (rarely) to 4 (most of the time) and has been used in numerous cross-cultural studies (e.g., Ryder et al., 2008). It measures symptoms such as loss of appetite, sleep problems, and loneliness. Internal reliability was excellent for the current sample $(\alpha=.91)$.

\section{Design and Analyses}

A pairwise correlation matrix was inspected for the first set of hypotheses. Bootstrapping analysis using the method of Preacher and Hayes (2008) was used to test for mediation related to the second hypothesis. Hierarchical multiple regression was used to test for the final moderation (interaction) hypothesis.

\section{Results}

SPSS Version 20 (IBM, Armonk NY, 2011) was used for the analyses. Missing data were not imputed. However, missing data were dealt with indirectly in the syntax by calculating mean item scores for participants for each measure, and including only those participants who completed at least 
Table 2. Correlation Matrix for Ethnic Density, Discrimination, Social Support, Acculturation, and Depression

\begin{tabular}{|c|c|c|c|c|c|c|}
\hline & $E D$ & PERDS & $C E S-D$ & MSPSS & QVIA-H & $Q V I A-F$ \\
\hline ED & - & $-.19 * 139$ & $-.17^{*}{ }_{138}$ & $-.03_{136}$ & $.00_{137}$ & $.06_{137}$ \\
\hline PERDS & & - & $.38^{* * *} 140$ & $-.23^{* *} 138$ & $-.10_{139}$ & $-.15_{139}$ \\
\hline CES-D & & & - & $-.29^{* *}{ }_{138}$ & $-.10_{138}$ & $-.15_{138}$ \\
\hline MSPSS & & & & - & $.19^{*}{ }_{137}$ & $.15_{137}$ \\
\hline QVIA-H & & & & & — & $.11_{139}$ \\
\hline QVIA-F & & & & & & - \\
\hline
\end{tabular}

Note. ED = perceived ethnic density; PERDS = Perceived Discrimination Scale; CES-D = Center for Epidemiologic Studies-Depression; MSPSS = Multidimensional Scale of Perceived Social Support; VIA-H = Vancouver Index of Acculturation-Heritage; VIA-F = Vancouver Index of Acculturation-French-Canadian Mainstream.

Pairwise $N$ s are provided in subscript.

$* p \leq .05 . * * p<.01$.

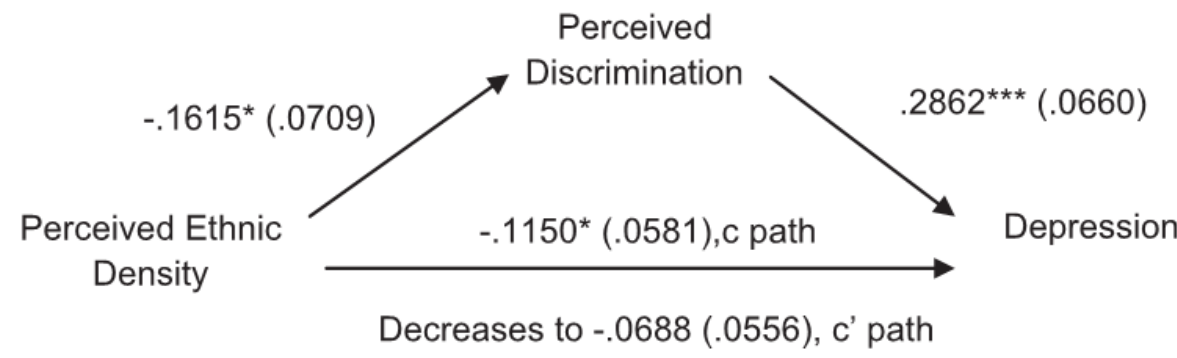

Figure 1. The relation between perceived ethnic density and depression mediated by perceived discrimination. Note. Indirect effect $=-.0462, S E=-.0263$. Percentile corrected 95\% CI $(-.1051$ to -.0016$)$ did not include (but were close to) zero. A bootstrap test with 5,000 re-samples was used (Preacher \& Hayes, 2008 ). $N=138$. $* p<.05, * * * p<.001$.

two thirds of each multi-item measure. The effect of univariate outliers was examined and the data was left unaltered. ${ }^{1}$

$$
1
$$

To preserve sample size, pairwise correlations were used. The bivariate correlation matrix (Table 2) indicated significant correlations between ethnic density and depression, $r$ $(136)=-.17, p=.05$, ethnic density and discrimination, $r$ $(137)=-.19, p=.03$, and between discrimination and depression, $r(138)=.38, p<.01$. Although social support correlated significantly with depression, $r(136)=-.29, p<.01$, it did not correlate with ethnic density, $r(134)=-.03, p=$ .73. Furthermore, neither heritage, $r(136)=-.10, p=.26$, nor mainstream acculturation, $r(136)=-.15, p=.07$, was significantly correlated with depression, or with ethnic den- sity, $r(135)=.00, p=.99$ for heritage and $r(135)=.06, p=$ .52 for mainstream acculturation.

A mediation model was tested using only perceived discrimination as the mediator between ethnic density and depression, but not social support, as social support did not correlate with ethnic density. Because age, gender, and household income were not associated with depression, background variables were not included in either the mediation

\footnotetext{
${ }^{1}$ Between one and three univariate outliers were identified (more than three but less than four $\mathrm{z}$-scores from the mean) for MSPSS, ED, QVIA-H, and age. However, after adjustment, they did not alter the pattern of results, and hence the original analyses are presented.
} 
Table 3. Hierarchical Multiple Regression of Depression (CES-D) on Ethnic Density, Discrimination, Social Support, Acculturation, and Ethnic Density Interactions

\begin{tabular}{|c|c|c|c|c|c|c|c|c|}
\hline \multirow[b]{2}{*}{ Variables } & \multicolumn{4}{|c|}{ Step 1} & \multicolumn{4}{|c|}{ Step 2} \\
\hline & $\beta$ & $s r$ & $t$ & $p$ & $\beta$ & $s r$ & $t$ & $p$ \\
\hline \multicolumn{9}{|l|}{ Main effects (Block 1) } \\
\hline${ }^{\mathrm{a}}$ Ethnic density (ED) & -.08 & -.07 & -0.93 & .36 & -.07 & -.07 & -0.89 & .38 \\
\hline Heritage Acculturation (VIA-H) & -.03 & -.03 & -0.37 & .71 & .20 & .12 & 1.53 & .13 \\
\hline French-Can. Acculturation (VIA-F) & -.07 & -.07 & -0.86 & .39 & -.11 & -.06 & -0.78 & .44 \\
\hline Social Support (MSPSS) & -.20 & -.19 & -2.39 & .02 & -.24 & -.17 & -2.10 & .04 \\
\hline Perceived Discrimination (PERDS) & .29 & .28 & 3.49 & .00 & .19 & .13 & 1.58 & .12 \\
\hline \multicolumn{9}{|l|}{ Ethnic density Interactions (Block 2) } \\
\hline${ }^{\mathrm{a}} \mathrm{ED} \times$ VIA-H & & & & & -.30 & -.18 & -2.25 & .03 \\
\hline${ }^{\mathrm{a}} \mathrm{ED} \times \mathrm{VIA}-\mathrm{F}$ & & & & & .07 & .04 & 0.49 & .62 \\
\hline${ }^{\mathrm{a}} \mathrm{ED} \times$ MSPSS & & & & & .08 & .05 & 0.67 & .50 \\
\hline${ }^{\mathrm{a}} \mathrm{ED} \times$ PERDS & & & & & .12 & .08 & 0.95 & .34 \\
\hline$R^{2}$ & $.19^{* * * *}$ & & & & $.23^{\text {**** }}$ & & & \\
\hline$R^{2}$ Change & & & & & .04 & & & \\
\hline
\end{tabular}

Note ED = perceived ethnic density; PERDS = Perceived Discrimination Scale; MSPSS = Multidimensional Scale of Perceived Social Support; VIA-H = Vancouver Index of Acculturation-Heritage; VIA-F = Vancouver Index of Acculturation-French-Canadian Mainstream; VIA-F = Vancouver Index of Acculturation-French Mainstream.

Standardized beta and semipartial correlations $(s r)$ are reported.

${ }^{\text {a }}$ To preserve group size in the regression, ethnic density was dichotomized into high (some or more, $n=85$ ) and low (none or hardly any, $n=50$ ) groups. The $n$ listed here are slightly less than in the text due to listwise selection in the regression. Age, gender, and household income did not correlate with depression and were therefore not entered in step 0 (block 0).

$* * * p<.001$. Italicized $p$ values in the table indicate significance.

or the moderation models. For the test of mediation $(N$ = 138), a bootstrap test with 5,000 re-samples was used (Preacher \& Hayes, 2008). This test has been found to be more powerful than other traditional tests of mediation, especially with small and non-normal distributions. An indirect effect (-.0462, standard error $[S E]=-.0263)$ of Ethnic Density to Perceived Discrimination to Depression was obtained. Percentile 95\% confidence intervals (CIs; -.1051 to -.0016), along with the other two CIs did not include (but were close to) zero, indicating statistically significant mediation (see Figure 1).

For the test of moderation (perceived ethnic density by heritage acculturation interaction for depression), a hierarchical multiple regression was conducted. Main effects were entered in Block 1 and interaction terms were added in Block 2. To preserve sample size, perceived ethnic density was collapsed into two groups: low (none or hardly any people from the participant's ethnic group; $n=53$ ) or high (some or more people from the participant's ethnic group; $n=90)$. Table 3 presents standardized beta $(\beta)$, semipartial correlations $(s r)$, $t$, and related probability $(p)$ values. Because the perceived ED effect is small in size, it was alone no longer significant after other variables were included in the regression model. Social support, $\beta=-.20, t(129)=-2.39, p=.02$, and perceived discrimination, $\beta=.29, t(129)=3.49$, $\mathrm{p}<.001$, were significant in step 1 , but only social support remained significant in step $2, \beta=-.24, t(125)=-2.10, p=.04$. Moreover, the ethnic density by heritage acculturation interaction was also significant in the second step, $\beta=-.30, t(125)=-2.25, p=$ .03 . Exploratory analyses revealed there was no interaction between ED and mainstream acculturation for depression, as well as between ED and social support and between ethnic density and discrimination.

The perceived ethnic density by heritage acculturation interaction for depression is presented in Figure 2, without including other variables. The crossover interaction suggested that the protective relation of heritage acculturation on depression scores was limited to those living in high ethnically dense neighborhoods, and reversed for those in low ethnically dense neighborhoods, leading to increased symptoms. 


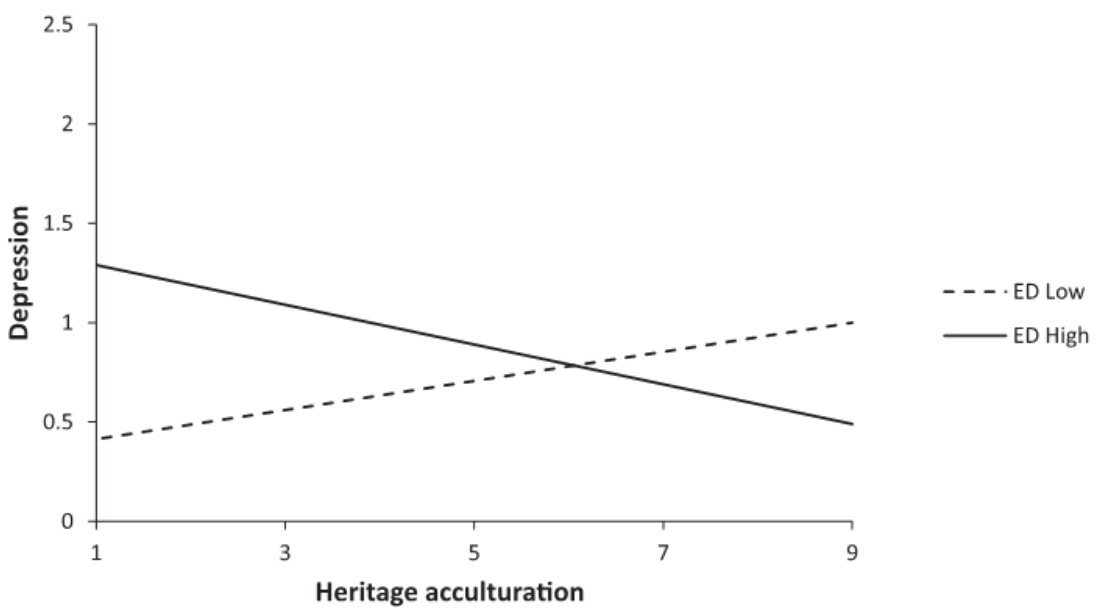

Figure 2. Interaction effect between ethnic density and heritage acculturation for depression without accounting for the effects of other variables.

Note. Mean item scores for depression and heritage acculturation are presented without accounting for other variables in the model.

This pattern was confirmed by examining simple effects for the high ED group, $\beta=-.27, t(83)=-2.56, \mathrm{R} 2=.07 ; p=$ .01 , and the low ED group, $\beta=.20, t(49)=1.41, R 2=.04$, $p=.16$. Thus, increasing heritage acculturation was related to significantly decreasing symptom scores for those in the higher density group, but there was a nonsignificant trend for increasing symptom scores for those in lower concentrated neighborhoods.

\section{Discussion}

The current study attempted to test discrimination and social support as mechanisms underlying the link between ethnic density and depression, and whether ethnic density could augment the protective relation between heritage acculturation and depression. Our predictions were partially supported. For the first set of hypotheses, perceived ED correlated negatively with depression and discrimination, but not positively with social support as we had expected. Discrimination was related positively to depression and negatively to social support, confirming the hypotheses. However, neither heritage nor mainstream acculturation was significantly correlated with depression. For the second hypothesis, the negative relation between ethnic density and depression was indeed mediated by perceived discrimination as anticipated, but not by social support. Finally, as expected, ethnic density moderated the heritage acculturation-depression relation. High heritage acculturation was protective in high ED contexts.

\section{Ethnic Density Effect Mediated by Discrimination}

This may be the first study showing a relation between perceived ethnic density and depression partially mediated by perceived discrimination. In contrast, Das-Munshi et al. (2010) did not find mediation effects between (objective) ethnic density and common mental disorders. Our study differed in that it used perceived ethnic density, focused on one disorder (i.e., depression vs. common mental disorders), used more graded or extensive measurements (e.g., continuous rather than dichotomous measures for depression, and a more detailed measure of discrimination), but a less sophisticated non-random sampling method. Subjective indicators may be better predictors of distress than objective ones (Christie-Mizell et al., 2003; Singh-Manoux et al., 2005), and it is possible that this finding generalizes to ethnic density as a predictor of mental health.

Although discrimination played a mediating role in the ethnic density-depression relation, it was surprising that social support did not correlate with ethnic concentration. This finding contrasts with suggestive qualitative (Whitley et al., 2006) and quantitative findings (Das-Munshi et al., 2010). Nevertheless, Das-Munshi et al. (2010) also failed to find an indirect effect of social support between ethnic density and common mental disorders. It is thus possible that social support may be less relevant to ethnic density than was previously believed, compared with the role of protecting new migrants from day-to-day discrimination. Social support may be obtained from other sources unrelated to the neighborhood, especially in the era of the Internet and Skype connec- 
tions, where immigrants can relatively easily keep in touch with friends and family in other countries (Ross, 2010). On the other hand, the current study may have been underpowered to find an indirect effect of social support and requires replication in larger samples. Future studies may also wish to explore the effect of long-distance social support on immigrant adjustment.

While immigrants may have more flexibility in choosing when and where they get their support, it may be more difficult to avoid experiencing discrimination in ethnically sparse neighborhoods. For example, because of convenience, immigrants may fall back on utilizing their neighborhood resources for grocery shopping, banking, recreational, and other activities. Frequently venturing beyond one's neighborhood for resources may be expensive and time consuming (Whitley et al., 2006), especially for student immigrants, some of whom may be living on more modest budgets. In such cases, discrimination may be more likely encountered in ethnically sparser neighborhoods, whose members may be less aware or tolerant of minority immigrant groups. However, ethnic density did not augment the effects of social support or mitigate the relation of discrimination on depression, which would also have been conceivable. This supports the specificity of the mechanism in the current sample: ethnic density was associated with less depression indirectly through reduced discrimination. Given that partial mediation was obtained, other potential mediators may need to be examined in future studies (e.g., social capital; Das-Munshi et al., 2009; Whitley et al., 2006).

\section{Matching Ethnic Density With Heritage Acculturation}

This study also expands upon the importance of studying acculturation research from an ecological "fit" perspective (see Birman et al., 2005; Miller et al., 2009). The significant crossover interaction indicated that a good fit or match (Schwartz et al., 2010) between ethnic density and heritage acculturation (i.e., high-high or possibly low-low) was related to less depression (Figure 2). In contrast, a lack of fit between acculturation and ethnic density (e.g., low-high) was related to higher levels of symptoms. A concordance between heritage acculturation and perceived neighborhood ethnic concentration may be beneficial for immigrants, where their beliefs and behaviors are reflected and reinforced in their environment. The current findings suggest that increased depressive symptoms may occur in individuals who do not identify with their heritage culture, but for whatever reason find themselves in an ethnically dense (i.e., "mismatched") neighborhood that on a day-to-day basis may not reflect such values and behaviors. While simple effects for the low ethnic density group were only trending (perhaps due to the smaller subsample), it seems possible that people who identified with their heritage culture but instead lived in a low ethnically dense environment may have experienced dissonance leading to an increased likelihood of depressive symptoms.

Miller and colleagues (2009) also obtained an interaction effect but it was less pronounced and limited to immigrant rather than ethnic concentration. However, these researchers examined Russian behavioral and American identity acculturation, with both variables having positive and negative relations with alienation, respectively, despite the moderating effect of (objective and overall) immigrant density. In contrast, the current study examined subjective ethnic concentration in a heterogeneous group of immigrant students, overall heritage acculturation (instead of behaviors or identity per se), and depression as an outcome variable rather than alienation. It is possible that extensive subjective measures of overall acculturation and depressive symptoms (rather than feelings of alienation) may be more sensitive to moderation effects, especially by another subjective rather than objective construct (i.e., ethnic density).

The current study's findings also differ somewhat from Birman and colleagues (2005), who found that mainstream acculturation (in this case American identity) interacted with ethnic density, where Russian high school students with greater American identity in high concentrated Russian neighborhoods in Chicago performed better at school. Their explanation was that there may have been a pull towards assimilation in the denser neighborhoods. However, this also took place in a different context with different samples and measures. Because Canada's policy leans less toward assimilation and more toward multiculturalism (see Berry, 2006b), where pluralism of heritage cultures is placed at a premium, there may be less of a push toward mainstream assimilation in the Montreal sample. This explanation is consistent with the finding that mainstream acculturation did not play a moderating role on ethnic density in our study while it did in Chicago (see also Miller et al., 2009). It is also curious that the Birman et al. (2005) participants reported more discrimination in ethnically dense neighborhoods, which goes against the findings here and in the general literature (e.g., Becares et al., 2009; Das-Munshi et al., 2010; Whitley et al., 2006). However, these discrepancies call for replication with the same measures in different contexts.

It was also unexpected that neither heritage nor mainstream acculturation would significantly correlate with depression scores in the current sample (cf. Ryder et al., 2000). However, we found heritage (but not mainstream) acculturation to be moderated by perceived ethnic density, and a recent study examining visible minority students in Montreal found heritage acculturation to be a significant predictor of adjustment (Ahmed et al., 2011). Other studies have found that mainstream acculturation to be a better predictor instead (Ryder et al., 2000; Zhang et al., 2010). These variations between samples and contexts are difficult to explain. While different ethnic groups do not adjust in the same way 
(Das-Munshi et al., 2010), as mentioned in the introduction, it is also possible that heritage acculturation may play a larger role with more recent immigrants in contexts where multiple mainstream groups exist and cultural segregation is relatively more pronounced, as in Montreal (see Balakrishnan et al., 2005; Bourhis et al., 2010). In turn, mainstream acculturation may be more relevant to mental health in less segregated contexts with only one dominant culture.

Finally, the findings add to the extant literature, suggesting that social support protects immigrants from depression (e.g., Mirsky, 2009; Ritsner et al., 1997), although social support was found to be independent of ethnic density in the current study, and that discrimination in minority groups is a risk factor for poor mental health outcomes (e.g., Kaspar \& Noh, 2003; Jung et al., 2007). Overall, the current student immigrant sample corroborated patterns obtained in community studies.

\section{Limitations, Strengths, and Future Directions}

Some notable limitations need to be addressed. First, we conveniently sampled a select heterogeneous and predominantly female undergraduate immigrant student population limiting generalizability. In contrast, Das-Munshi et al. (2010) randomly sampled specific ethnic groups in the community and noted that the ethnic density effect may be operating differently in the various subgroups. On the other hand, the patterns of associations obtained in the current sample are similar to those found in community groups (e.g., Becares et al., 2009; Mirsky, 2009; Noh \& Kasper, 2003; Jung et al., 2007), suggesting the sample may serve as a useful model.

A second limitation is the correlational design, which is obscuring the direction of effect. The term ethnic density effect implies that a higher ethnic concentration somehow leads to less distress but other explanations are also possible. For example, once people become depressed in neighborhoods with a greater ethnic concentration, they may tend to move to more ethnically sparse neighborhoods. Longitudinal studies may help resolve directions of causality questions by regularly keeping track of immigrant movement between neighborhoods and symptom scores. Third, ethnic concentration was perceived, in the absence of comparison objective measurement (cf. Strafford et al., 2009) and other neighborhood level variables that may have acted as potential mediators (e.g., neighborhood disorder and capital). Objective ethnic density was not measured in the current sample because of its considerable heterogeneity. However, subjective and objective ethnic density have previously been found to be moderately correlated (Stafford et al., 2009), and given the positively skewed distribution of responses in the current ethnically diverse sample, perceived ethnic concentration may have been a fairly accurate marker of objective density.

Further research will be needed to compare the predictive power of subjective compared to objective ethnic den- sity. Finally, it is possible that ethnic density also plays a role in protecting immigrants in other non-neighborhood settings (e.g., workplace, academic life) that have yet to be evaluated. We are currently collecting more extensive community data in the Montreal area, which is less gender-biased and more representative in socioeconomic status, including the oversampling of the Russian-speaking immigrant community to overcome some of the above shortcomings.

Despite its limitations, the current study also makes some notable contributions. First, although a potential sampling concern, the ethnic density effect prior to this study has to our knowledge not yet been closely examined with a diverse immigrant student sample. Second, it is an addition to the very few studies that have sought to empirically unpack the ethnic density effect, and perhaps the first study to unpack the perceived ethnic density effect. This study is to our knowledge the first to show a significant mediation effect of discrimination partly explaining the relation between perceived ethnic density and depression. This is a notable contribution supporting the utility of subjective indicators, which may be better predictors than objective ones (e.g., social status on health; Singh-Manoux et al., 2005).

The current study also makes a contribution to acculturation research that has to date generally been studied without much attention to contextual variables. Given the perceived ethnic density-acculturation interaction, the current findings along with those of others (Birman et al., 2005; Miller et al., 2009) may motivate psychologists to incorporate neighborhood level data such as ethnic concentration when studying processes of acculturation and adjustment.

Our findings also have clinical and policy implications in need of further investigation. Primary prevention programs may utilize and evaluate educational and sensitizing interventions focusing on reducing discrimination in ethnically dispersed neighborhoods (see also Birman et al., 2005; Miller et al., 2009). The effectiveness of clinical ecological interventions that encourage distressed immigrants to live in or more frequently visit neighborhoods concordant with their acculturation style may also generate interesting findings, but should not be used to foster segregation and undermine primary prejudice prevention programs.

Currently, some clinical textbooks suggest the importance of assessing unidimensional acculturation (i.e., heritage to mainstream on one scale) for treatment planning (e.g., Paniagua et al., 2005). However, evaluating bidimensional acculturation may be a more useful approach (Ryder et al., 2000), assessed in combination with patient ecology (e.g., perceived neighborhood ethnic concentration) to better contextualize challenges and resilience related to mental health. For example, clinicians in community settings treating depressed immigrants may hypothesize a "mismatch" between heritage acculturation level and perceived ethnic density and consider testing such an assumption with available 
measures. Investigation of such an approach could advance the clinical utility of acculturation scales.

Summary. The current study found that the negative relation between perceived ethnic density and depression was mediated by reduced perceived discrimination, but not by increased social support. The relation between heritage acculturation and reduced depression applied for those residing in high but not low ethnic density neighborhoods. These findings build on the recently emerging literature attempting to explain the mechanisms of the ethnic density effect, and highlight the importance of fit between acculturation and ecological factors for improved mental health. The clinical utility of assessing perceived ethnic density in the context of bicultural acculturation with immigrant clients needs to be further explored.

\section{References}

Ahmed, R., Jurcik, T., \& Ryder, A. G. (2011). The role of attachment, acculturation, and social support in predicting the psychosocial adjustment of visible immigrants: Towards an integrative model. Poster presented at the 72nd annual convention of the Canadian Psychological Association, Toronto, ON.

Balakrishnan, T. R., Maxim, P., \& Jurdi, R. (2005). Social class versus cultural identity as factors in the residential segregation of ethnic groups in Toronto, Montreal and Vancouver for 2001. Canadian Studies in Population, 32, 203-227.

Bècares, L., Nazroo, J., \& Stafford,M. (2009). The buffering effects of ethnic density on experienced racism and health. Health $\mathcal{F}$ Place, 15, 700-708. doi:10.1016/j.healthplace.2008.10.0

Bècares, L., Nazroo, J., \& Stafford, M. (2011). The ethnic density effect on alcohol use among ethnic minority people in the UK. Journal of Epidemiology and Community Health, 65, 20-25. doi:10.1136/jech.2009.087114

Berry, J. W. (1997). Immigration, acculturation and adaptation. Applied Psychology: An International Review, 46, 5-68. doi:10.1080/026999497378467

Berry, J. W. (2006a). Stress perspectives on acculturation. In D. Sam \& J. W. Berry (Eds.), The handbook of acculturation psychology (pp. 43-57). Cambridge, UK: Cambridge University Press.

Berry, J. W. (2006b). Contexts of acculturation. In D. Sam \& J. W. Berry (Eds.), The handbook of acculturation psychology (pp. 27-42). Cambridge, UK: Cambridge University Press.

Birman, D., Trickett, E., \& Buchanan, R. M. (2005). A tale of two cities: Replication of a study on the acculturation and adaptation of immigrant adolescents from the former Soviet Union in a different community context. American Journal of Community Psychology, 35, 87-101. doi:10.1007/s10464-005-1891-

Bourhis, R. Y., Montaruli, E., El-Geledi, S., Harvey, S. P., \& Barrette, G. (2010). Acculturation in multiple host community settings. Journal of Social Issues, 66, 780_802. doi:10.1111/j.1540-4560.2010.01675.x

Boydell, J., van Os, J., McKenzie, K., Allardyce, J., Goel, R., McCreadie, R. G., \& Murray, R. M. (2001). Incidence of schizophrenia in ethnic minorities in London: Ecological study into interactions with environment. British Medical Journal, 323, doi:10.1136/bmj.323.7325.1336

Christie-Mizell, C. A., Steelman, L. C., \& Jennifer, S. (2003). Seeing their surroundings: The effects of neighborhood setting and race on maternal distress. Social Science Research, 32, 402-428. doi:10.1016/S0049-089X(03)00014-0 Costigan, C. L., \& Su, T. F. (2004). Orthogonal versus linear models of acculturation among immigrant Chinese Canadians: A comparison of mothers, fathers, and children. International Journal of Behavioral Development, 28, 518-527. doi:10.1080/01650250444000234

Das-Munshi, J., Becares, L., Dewey, M. E., Stansfeld, S. A., \& Prince, M. J. (2010). Understanding the effect of ethnic density on mental health:Multi-level investigation of survey data from England. British Medical Journal, 34, 1—9. doi:10.1136/bmj.c5367

Flannery, W. P., Reise, S. P., \& Yu, J. (2001). An empirical comparison of acculturation models. Personality and Social Psychology Bulletin, 27, 1035-1045. doi:10.1177/0146167201278010

Gordon, M. M. (1964). Assimilation in American life. New York, NY: Oxford University Press.

Immigration et communautés culturelles Québec. (2012). Partenaires en francisation. Retrieved from http://www.immigration-quebec.gouv.qc.ca/fr/partenaires/pa rtenaires-francisation/

Jung, E., Hecht, M. L., \& Wadsworth, B. C. (2007). The role of identity in international students' psychological well-being in the United States: A model of depression level, identity gaps, discrimination, and acculturation. International Journal of Intercultural Relations, 31, 605-624. doi:10.1016/j.ijintrel.2007.04.001

Malzberg, B. (1964). Mental disease in Canada, 1950-1952: A study of comparative incidence of mental disease among those of British and French origin. Albany, NY: Research Foundation for Mental Hygiene.

Miller, A. M., Birman, D., Zenk, S., Wang, E., Sorokin, O., \& Connor, J. (2009). Neighborhood immigrant concentration, acculturation, and cultural 
alienation in former Soviet immigrant women. Journal of Community Psychology, 37, 88-105. doi:10.1002/jcop.20272

Mirsky, J. (2009). Mental health implications of migration: A review of mental health community studies on Russian-speaking immigrants in Israel. Social Psychiatry and Psychiatric Epidemiology, 44, 179-187. doi:10.1007/s00127-008-0430-1

Neeleman, J., Wilson-Jones, C., \& Wessely, S. (2001). Ethnic density and deliberate self harm; a small area study in southeast London. Journal of Epidemiology and Community Health, 55, 85-90. doi:10.1136/jech.55.2.85

Noh, S., \& Kaspar, V. (2003). Perceived discrimination and depression: Moderating effects of coping, acculturation, and ethnic support. American Journal of Public Health, 93, 232-238. doi:10.2105/AJPH.93.2.232

Paniagua, F. A. (2005). Assessing and treating culturally diverse clients: A practical guide (3rd ed.). Thousand Oaks, CA: Sage

Preacher, K. J., \& Hayes, A. F. (2008). Asymptotic and resampling strategies for assessing and comparing indirect effects in multiple mediator models. Behavior Research Methods, 40, 879-891. doi:10.3758/BRM.40.3.879

Radloff, L. S. (1977). The CES-D scale: A self-report depression scale for research in the general population. Applied Psychological Measurement, 1, 385-401. doi:10.1177/014662167700100306

Ritsner, M., Ponizovsky, A., \& Ginath, Y. (1997). Changing patterns of distress during the adjustmentnof recent immigrants: A 1-year follow-up study. Acta Psychiatrica Scandinavica, 95, 494-499. doi:10.1111/j.1600-0447.1997.tb10137.x

Ross, A. (2010). Interconnected immigrants in the information society. In A. Alonsi \& P.J. Oiarzabal (Eds.), Diasporas in the new media age: Identity, politics and community (pp. 19-38). Reno, NV: University of Nevada Press.

Rudmin, F. (2003). Critical history of acculturation psychology of assimilation, separation, integration, and marginalization. Review of General Psychology, 7(1), 3-37.

Rudmin, F. W. (2009). Constructs, measurements and models of acculturation and acculturative stress. International Journal of Intercultural Relations, 33, 106-123. doi:10.1016/j.ijintrel.2008.12.001

Ryder, A. G., Alden, L. E., \& Paulhus, D. L. (2000). Is acculturation unidimensional or bidimensional?: Ahead-to-head comparison in the prediction of demographics, personality, self-identity, and adjustment. Journal of Personality and Social Psychol- ogy, 79, 49-65. doi:10.1037/0022-3514.79.1.49

Ryder, A. G., \& Dere, J. (2010). Review: Cambridge handbook of acculturation psychology. Journal of Cross-Cultural Psychology, 41, 278-283.

Ryder, A. G., Yang, J., Zhu, X., Yao, S., Yi, J., Heine, S. J., \& Bagby, R. M. (2008). The cultural shaping of depression: Somatic symptoms in China, psychological symptoms in North America? Journal of Abnormal Psychology, 117, 300-313. DOI:10.1037/0021-843X.117.2.300

Sam, D. (2006). Acculturation and health. In D. Sam \& J. W. Berry (Eds.), The handbook of acculturation psychology (pp. 452-468). Cambridge, UK: Cambridge University Press.

Schwartz, S. J., Unger, J. B., Zamboanga, B. L., \& Szapocznik, J. (2010). Rethinking the concept of acculturation: Implications for theory and research. American Psychologist, 65, 237-251. doi:10.1037/a0019330.

Singh-Manoux, A., Marmot,M. G., \& Adler, N. E. (2005). Does subjective social status predict health and change in health status better than objective status? Psychosomatic Medicine, 67(6), 855-861. doi:10.1097/01.psy.0000188434.52941.a0

Stafford, M., Bécares, L., \& Nazroo, J. (2009). Objective and perceived ethnic density and health: findings from a UK general population survey. American Journal of Epidemiology, 170, 484-493. doi:10.1093/aje/kwp160

Stafford, M., Bécares, L., \& Nazroo, J. (2010). Racial discrimination and health: Exploring the possible protective effects of ethnic density. In J. Stillwell \& M. van Ham (Eds.), Ethnicity and Integration. Retrieved from: http://www.uptap.net/wordpress/wp-content/ uploads/2010/08/uptap-findings-stafford-jul10.pdf

Stafford, M., Newbold, B. K., \& Ross, N. (2011). Psychological distress among immigrants and visible minorities in Canada: A contextual analysis. International Journal of Social Psychiatry, 57, 428-441. doi:10.1177/0020764010365407

Statistical Package for the Social Sciences (SPSS, Version 20 for Windows). Rel. 09.14. 2011. Armonk, NY: IBM.

Whitley, R., Prince, M., McKenzie, K., \& Stewart, R. (2006). Exploring the ethnic density effect: A qualitative study of a London electoral ward. International Journal of Social Psychiatry, 52, 376-391. doi:10.1177/0020764006067239

Zhang, J., Mandl, H., \& Wang, E. (2010). Personality, acculturation, and psychosocial adjustment of Chinese international students in Ger- 
many. Psychological Reports, 107, 511-525. doi:10.2466/07.09.11.17.PR0.107.5.511-525

Zimet, G. D., Dahlem, N. W., Zimet, S. G., \& Farley, G. K.
(1988). The Multidimensional Scale of Perceived Social Support. Journal of Personality Assessment, 52, 30—41. doi:10.1207/s15327752jpa5201_2 